\title{
Enhanced efficiency of the second harmonic inhomogeneous component in an opaque cavity
}

\author{
V. Roppo, ${ }^{1,2, *}$ F. Raineri,,${ }^{3,4}$ R. Raj, ${ }^{3}$ I. Sagnes, ${ }^{3}$ J. Trull, ${ }^{1}$ R. Vilaseca, ${ }^{1}$ M. Scalora, ${ }^{2}$ and C. Cojocaru ${ }^{1}$ \\ ${ }^{1}$ Departament de Física i Enginyeria Nuclear, Universitat Politècnica de Catalunya, Rambla Sant Nebridi, o8222 Terrassa, Spain \\ ${ }^{2}$ Charles M. Bowden Research Facility, U.S. Army RDECOM, Redstone Arsenal, Alabama 35803, USA \\ ${ }^{3}$ Laboratoire de Photonique et de Nanostructures, Route de Nozay 91460 Marcoussis, France \\ ${ }^{4}$ Université Paris-Diderot, 75205 Paris Cedex 13, France \\ ${ }^{*}$ Corresponding author: vito.roppo@upc.edu
}

Received October 22, 2010; revised March 31, 2011; accepted April 13, 2011;

posted April 18, 2011 (Doc. ID 137052); published May 9, 2011

In this Letter, we experimentally demonstrate the enhancement of the inhomogeneous second harmonic conversion in the opaque region of a GaAs cavity with efficiencies of the order of $0.1 \%$ at $612 \mathrm{~nm}$, using $3 \mathrm{ps}$ pump pulses having peak intensities of the order of $10 \mathrm{MW} / \mathrm{cm}^{2}$. We show that the conversion efficiency of the inhomogeneous, phase-locked second harmonic component is a quadratic function of the cavity factor Q. () 2011 Optical Society of America

OCIS codes: $190.2620,190.5530$.

Second harmonic generation (SHG) [1-3] is perhaps the most studied nonlinear optical phenomenon. When a fundamental field (FF) impinges on a $\chi^{(2)}$ material, the resulting polarization is a quadratic function of the field. The generated second harmonic (SH) signal has a carrier frequency that doubles the FF one. Even though the general solution of a problem that includes dispersion and absorption is rather complicated, it is possible to derive approximate solutions that elucidate shape and behavior of the generated SH [1]. The general solution of an inhomogeneous (INH) set of differential equations consists of two parts: the first is the solution of the homogeneous (HOM) equations, the second is a particular solution of the INH system. The former (HOM SH) has a well-known expression, and its behavior and efficiency have been investigated extensively, especially under phase matching conditions, i.e., the fields propagate with the same phasevelocity [늠] . The latter (INH SH) does not have an exact or unique form. The INH solution manifests itself under phase-mismatched conditions with conversion efficiencies smaller than the phase-matched case. This is why the study of the INH SH has generated very little interest.

During the last few decades, relatively few studies have reported this twofold nature of the generated $\mathrm{SH}$ signal, aspect that gives rise to Maker fringes $[7,8]$ and a double-peaked SH signal shape [9-11]. Nevertheless, efforts have remained focused on studying phasematched SHG to ensure maximum conversion efficiency. As we will see later [12] , in uniform media, only the HOM SH component can be enhanced, a situation that makes the INH SH component more difficult to observe.

This relative lack of knowledge regarding the dynamics of pulses generated in the mismatched regime and the availability of laser sources with ever decreasing pulse durations made it necessary that a systematic investigation of the INH SH be undertaken $[12,13]$. Since it is not an easy task to glean its properties from the implicit form of the analytical solution, one has to rely on numerical studies. In [12,13], the dynamics of the INH SH component is discussed in some detail. This component of the $\mathrm{SH}$ is always present during propagation and travels bound to the FF with the same velocity. Once generated within the first few coherence lengths, as the pump pulse traverses the entry interface, the SH energy clamps with no further energy exchange with the pump pulse. Even under phase-mismatched conditions, the carrier wave vector of the trapped INH SH pulse is always twice that of the $\mathrm{FF}$, leading to a phase locking phenomenon that is not unlike the phase locking that occurs between two coupled harmonic oscillators that have different resonant frequencies. A simple way to sum up these characteristics is to state that the INH SH component (oscillator 1) effectively "reads" the same complex index of refraction as the FF (oscillator 2). Thus, pulse propagation in long crystals leads to the identification of the INH SH and rapid walk-off of the HOM component, which proceeds unencumbered in the media decoupled from the pump field, experiencing the nominal dispersion expected at the SH frequency.

Experimental and numerical verifications of this phenomenon have been reported in [13-15]. In $[13,14]$, the delay in collinear propagation and the different refraction angles that appear due to the different indices of refraction experienced by the two beams were measured for both the INH SH and HOM SH components. In [15], it was shown that the imaginary part of the index of refraction of the locked INH SH pulse was identical to that of the pump. A FF beam was tuned in a transparency region $(1300 \mathrm{~nm})$ of a GaAs slab and generated SH (650 nm) and third harmonic (TH) $(433 \mathrm{~nm})$ signals deep in the absorption region. The absorption band edge for GaAs is located at $900 \mathrm{~nm}$. As expected, the HOM SH is quickly absorbed while the INH SH component traverses the entire sample without being absorbed. This effect can be understood as inhibition of absorption at the harmonic wavelength for the INH component. Although one might be tempted to think that the INH SH is constantly being replenished by the pump, our simulations show, and experiments suggest $[15,16]$, that pulses travel very long distances (hundreds of thousands of absorption lengths) 
without gain or loss. If the pump were indeed replenishing its harmonics, it would most certainly be depleted far from the exit side. This depletion would be visible in the numerical simulations, even if the efficiency of the process is low. More numerical details can be found in [17].

These findings present us with new opportunities for at least two reasons. First, one can extend the useful spectral range for harmonic generation well below the material absorption edge, including UV and extreme UV (X-UV) frequencies. Second, as absorption removes the HOM component, one may deeply study the INH SH component only. Unfortunately, the conversion efficiency of the INH SHG from a single interface of semiconductorlike materials is rather low (of order $10^{-9}-10^{-10}$ ), as reported in $[7,15]$. We thus pose the following question: is it possible to enhance SHG in the phase mismatched regime using ordinary laser sources and materials, up to a level that rivals SHG from phase-matched or quasi-phase-matched materials? The answer is affirmative if we take advantage of the "induced transparency" of the material for the INH SH component under resonant conditions.

In [18], the behavior of the INH SH in a cavity environment was theoretically predicted. The GaAs cavity was designed to be resonant only at the FF frequency. $\mathrm{SH}$ and TH signals were not resonant due to material dispersion and absorption. The results showed that the HOM $\mathrm{SH}$ is mostly absorbed, while the INH SH becomes localized inside the cavity with an effective index that matched the FF index of refraction. This localization, together with the localization of the FF field and the resulting good overlap between the fields, leads to improvements of the SH conversion efficiency. The effect was partially experimentally shown in [19], where a low- $Q$ cavity $(Q \sim 10)$ improved the efficiency by 2 orders of magnitude, up to $\sim 10^{-7}$. It was estimated that the $\mathrm{SH}$ efficiency is proportional to $Q^{2}$, based on Fermi's golden rule. In what follows, we present results that confirm that this simple relation is a practical tool to predict conversion efficiencies of the INH SH component, in good agreement with our experimental results. At the same time, the efficiency of the INH SHG may be of order $0.1 \%$ in a cavity made of an absorptive material.

We have designed four different cavities made of a $350 \mathrm{~nm}$ thick GaA slabs sandwiched between two distributed Bragg mirrors having different reflectivities (inset Fig. 1). The mirrors were designed with $4,6,8$, and 10 pairs of $\mathrm{SiO}_{2}(120 \mathrm{~nm}) / \mathrm{Si}_{3} \mathrm{~N}_{4}(210 \mathrm{~nm})$ layers, respectively. Each of these samples has a resonance centered at the same wavelength, but they have different spectral width due to their different reflectivities and $Q$ factors. Resonance shape and width were ascertained experimentally by illuminating the samples with a femtosecond pulse, and are shown in Fig. 1. The resonances are centered at $\lambda=1225 \mathrm{~nm}$ and have FHMW of $9.3,2.6,1.2$, and $0.32 \mathrm{~nm}$. The corresponding cavity $Q \mathrm{~s}(Q \equiv \lambda / \Delta \lambda)$, are $125,350,1200$, and 3830 , respectively.

The difference between this sandwich and a freestanding GaAs etalon [18] is that here the internal field is hundreds of times more intense than the incident field. The stack is transparent at the FF wavelength but absorbs at the SH wavelength. For the cavity used in our experiments, we measured a linear transmission of $5 \%$. This

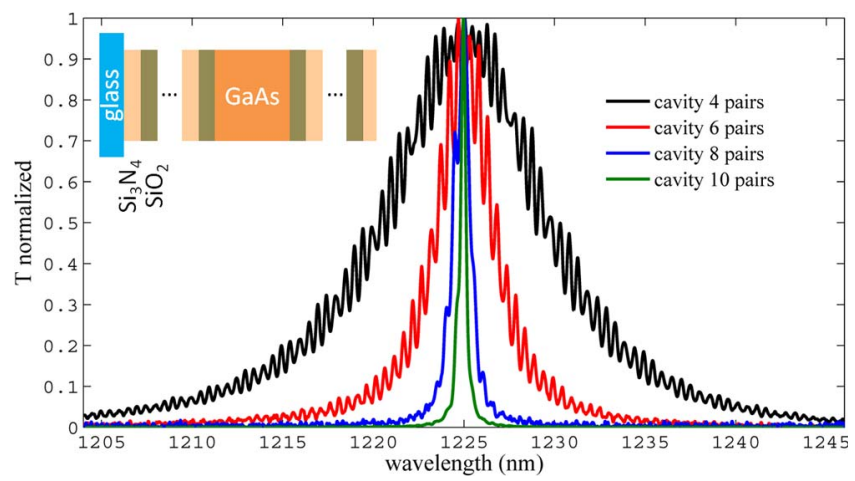

Fig. 1. (Color online) Samples' transmission spectra. The curves are obtained from the transmission spectra of a femtosecond laser. The fast oscillations are due to the multiple reflections inside the $1 \mathrm{~mm}$ glass substrate. The transmittance scale is normalized for graphical reason. The peaks reach $0.74,0.34$, 0.26 , and 0.06 for the $10,8,6$, and 4 couple mirrors, respectively. As a reference, the FF field spectrum is $\sim 2 \mathrm{~nm}$ FWHM. Inset: sample scheme.

is in good agreement with our numerical results. This means that the detected SH signal is $\geq 95 \%$ in the INH component, and less than $5 \%$ is the residual HOM signal, as absorption limits propagation of the HOM portion.

The source of the FF pulse $(\lambda=1225 \mathrm{~nm})$ is a tunable optical parametric amplifier, slightly focused down to about $500 \mu \mathrm{m}$ at the sample. Its duration was fixed at $3 \mathrm{ps}$, and the incident intensity was about $10 \mathrm{MW} / \mathrm{cm}^{2}$. The transmitted FF signals were sent to a spectrometer connected to a cooled AlGaAs camera. The SH signals were recorded with a $\mathrm{Si}$ detector. The incident angle was $10^{\circ}$ to take advantage of the nonzero $\chi^{(2)}$ in that direction $\left(\chi^{(2)}=0\right.$ at $\left.0^{\circ}\right)$. The setup is sketched in Fig. 3 . The SH signal was normalized to the part of the input FF energy that finds its way inside the cavity. To account for the spectral width of the pulse, for each sample, we calculated the overlap between the FF pulse and the cavity spectra.

After characterizing the cavity resonances, pulses were shortened to about $80 \mathrm{fs}$. Pulses this short can scan the transmission of the sample. By multiplying this spectrum for the spectrum of the $3 \mathrm{ps}$ pulse used during the experiment, we can estimate the FF energy that actually penetrated the cavity. For each sample, we measured the $\mathrm{SH}$ output power as a function of the FF power coupled

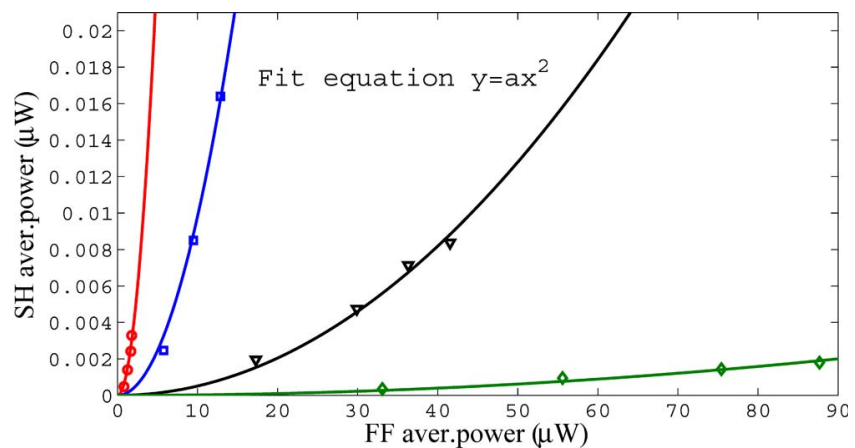

Fig. 2. (Color online) $\mathrm{SH}$ average power versus $\mathrm{FF}$ average power for all samples. The continuous curves are the quadratic fits of the experimental data (circles). The laser output power is always in the same range, but the effective powers inside the cavities depend on the resonance width. 


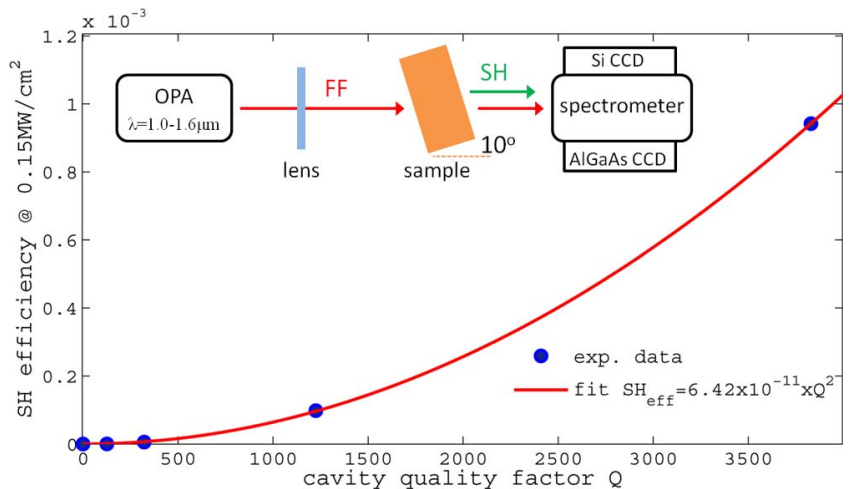

Fig. 3. (Color online) SH efficiency versus cavity- $Q$ factor, experimental data, and quadratic fit. Absorption at the $\mathrm{SH}$ wavelength leaves the INH component intact. Inset: experimental setup.

into the cavity (Fig. 2). The internal FF power becomes smaller for higher $\bar{Q}$ s due to the smaller resonance bandwidth. Simultaneously, the enhancement of the FF field increases with higher $Q \mathrm{~s}$. As shown in the figure, the SH follows a regular quadratic pattern. This proves that two-photon absorption or other nonlinear effects are not taking part in the generation in any appreciable measure.

Finally we calculated the efficiency of the INH SH conversion versus cavity- $Q$, shown in Fig. 3. To compare the efficiency obtained with each of the four samples, we chose the same value of the FF intensity, namely $0.15 \mathrm{MW} / \mathrm{cm}^{2}$ (this corresponds roughly to the last experimental point for the 10-pair mirrors in Fig. 2). This value is low enough to avoid two-photon absorption for all samples. We extrapolated the SH power corresponding to this $\mathrm{FF}$ intensity from the results shown in Fig. 2. The circles show the efficiencies obtained with each sample; the solid curve is a quadratic fit of the data. The first circle marks the value that corresponds to the bulk emission [15]. The results are thus in good agreement with the general estimates made in [19]. The figure shows that it is possible to achieve efficiencies close to $0.1 \%$ using external pump peak intensities of order $10 \mathrm{MW} / \mathrm{cm}^{2}$, which for $3 \mathrm{ps}$ incident pulses leads to $0.15 \mathrm{MW} / \mathrm{cm}^{2}$ inside the cavity. There is real potential for pump depletion with relatively low intensities (tens of $\mathrm{MW} / \mathrm{cm}^{2}$ ), provided incident pulses resolve the resonance. In our case, the FF peak power was kept low to avoid two-photon absorption, which can be considerable in GaAs at these wavelengths. Beyond this, the INH SH behavior is general and devices may be designed by choosing materials like polymers, which can outperform GaAs given the potential for much higher nonlinear coefficients [20]. Finally, these results are easily extended to TH generation, as shown in $[15,18,19]$. In this particular case, the cavity was not optimize for TH generation, as it happened to be tuned inside the bandgap of the stack.

In conclusion, we experimentally demonstrated $\mathrm{SH}$ conversion efficiencies that approach $0.1 \%$ in an opaque GaAs cavity using incident pump intensities of order
$10 \mathrm{MW} / \mathrm{cm}^{2}$. We have also shown that the conversion efficiency of the INH, phase-locked, SH component is a quadratic function of cavity- $Q$, an indication of the enhancement of the FF inside the cavity. These results show that high conversion efficiencies can be obtained in phase-mismatched materials in ways that may also be practical. In addition, with the right materials it becomes possible to exploit new wavelength ranges, e.g. UV-X-UV, using GaP or GaN.

This work was partially supported by the Spanish Government (FIS2008-06024-C03-02), the Catalan Government (2009 SGR 1168), and the Army Research Office (W911NF-10-2-0105). The authors thank D. Chouteau for his contributions in sample fabrication.

\section{References}

1. N. Bloembergen and P. S. Pershan, Phys. Rev. 128, 606 (1962).

2. J. A. Armstrong, N. Bloembergen, J. Ducuing, and P. S. Pershan, Phys. Rev. 127, 1918 (1962).

3. P. A. Franken, A. E. Hill, C. W. Peters, and G. Weinreich, Phys. Rev. Lett. 7, 118 (1961).

4. W. H. Glenn, IEEE J. Quantum Electron. 5, 284 (1969).

5. M. M. Fejer, Phys. Today 47, 25 (1994).

6. N. Bloembergen and A. J. Sievers, Appl. Phys. Lett. 17, 483 (1970).

7. P. D. Maker, R. W. Terhune, M. Nisenoff, and C. M. Savage, Phys. Rev. Lett. 8, 21 (1962).

8. J. Jerphagnon and S. K. Kurtz, J. Appl. Phys. 41, 1667 (1970).

9. L. D. Noordam, H. J. Bakker, M. P. de Boer, and H. B. van Linden van den Heuvell, Opt. Lett. 15, 1464 (1990).

10. W. Su, L. Qian, H. Luo, X. Fu, H. Zhu, T. Wang, K. Beckwitt, Y. Chen, and F. Wise, J. Opt. Soc. Am. B 23, 51 (2006).

11. T. B. Kristensen and K. Pedersen, Opt. Commun. 233 , 219 (2004).

12. V. Roppo, M. Centini, C. Sibilia, M. Bertolotti, D. de Ceglia, M. Scalora, N. Akozbek, M. J. Bloemer, J. W. Haus, O. G. Kosareva, and V. P. Kandidov, Phys. Rev. A 76, 033829 (2007).

13. M. Mlejnek, E. M. Wright, J. V. Moloney, and N. Bloembergen, Phys. Rev. Lett. 83, 2934 (1999).

14. E. Fazio, F. Pettazzi, M. Centini, M. Chauvet, A. Belardini, M. Alonzo, C. Sibilia, M. Bertolotti, and M. Scalora, Opt. Express 17, 3141 (2009).

15. M. Centini, V. Roppo, E. Fazio, F. Pettazzi, C. Sibilia, J. W. Haus, J. V. Foreman, N. Akozbek, M. J. Bloemer, and M. Scalora, Phys. Rev. Lett. 101, 113905 (2008).

16. V. Roppo, J. V. Foreman, N. Akozbek, M. A. Vincenti, and M. Scalora, Appl. Phys. Lett. 98, 111105 (2011).

17. V. Roppo, "Nonlinear frequency conversion under general phase mismatched conditions: the role of phase locking and random nonlinear domains," Ph.D. dissertation (Universitat Politecnica de Catalunya, 2011).

18. V. Roppo, C. Cojocaru, J. Trull, R. Vilaseca, and M. Scalora, Waves Random and Complex Media 20, 319 (2010).

19. V. Roppo, C. Cojocaru, F. Raineri, G. D'Aguanno, J. Trull, Y. Halioua, R. Raj, I. Sagnes, R. Vilaseca, and M. Scalora, Phys. Rev. A 80, 043834 (2009).

20. S. Bergfeld and W. Daum, Phys. Rev. Lett. 90, 036801 (2003). 